\title{
Azithromycin Protects Oligodendrocyte Progenitor Cells against Lipopolysaccharide- Activated Microglia-Induced Damage
}

\author{
Sumana Ramarao Yi Pang Kathleen Carter Abhay Bhatt \\ Division of Newborn Medicine, Department of Pediatrics, University of Mississippi Medical Center, \\ Jackson, MS, USA
}

\section{Keywords}

Encephalopathy of prematurity · Neuroinflammation . Neuroprotection - Very low-birth weight infants · White matter injury

\section{Abstract}

Oligodendrocyte progenitor cells (OPC) are the primary cellular targets of brain white matter injury (WMI) in very lowbirth weight (VLBW) infants. Microglia plays a significant role in inflammation-induced WMI. Our previous study showed that lipopolysaccharide (LPS)-induced OPC damage is mediated by activated microglia in vitro. We hypothesized that azithromycin (AZ) could protect OPCs against LPS-induced cytotoxicity by blocking microglial activation. Highly enriched primary rat microglia and OPCs were treated with LPS. There were 4 groups: control, LPS + Veh, AZ, and LPS + AZ. Microglia conditioned medium (MCM) was used to determine inflammatory cytokines by enzyme-linked immunosorbent assay or subsequent treatment of OPCs. We found that AZ significantly suppressed TNF- $a$, IL-1 $\beta$, and IL- 6 in LPS+Veh-treated-microglial MCM and blocked microglial nuclear factor-kB p65 nuclear translocation. AZ prevented LPS-MCM-induced OPC death and improved OPC survival as measured by activated caspase- 3 immunostaining and XTT assay, respectively. AZ ameliorated LPS-MCM-induced differentiation arrest and myelin basic protein deficit in oligodendrocytes. Our data suggest that $A Z$ is a potent inhibitor for microglia activation and may hold the therapeutic potential for WMI in VLBW infants.

(C) 2021 The Author(s)

Published by S. Karger AG, Basel

\section{Introduction}

There is a critical lack of knowledge for the prevention and treatment of white matter injury (WMI), the major form of brain injury in premature infants that is associated with a spectrum of motor, cognitive, visual, socialbehavioral, attention, and learning disabilities in $25-50 \%$ of very low birth weight $(<1,500 \mathrm{~g})$ survivors [1-3]. WMI is characterized by initial loss and/or dysmaturation of oligodendrocyte (OL) progenitor cells (OPCs) followed by subsequent hypomyelination and dysmaturation events of neuroaxonal structures evolving over a prolonged period [1]. OPCs are the dominant OL lineage population at 24-32 weeks of gestation, which is the highrisk window for developing brain WMI [4]. Mounting

karger@karger.com www.karger.com/dne

Karger $\stackrel{\text { ' }}{=}$

BOPEN ACCESS
(C) 2021 The Author(s)

Published by S. Karger AG, Basel

This is an Open Access article licensed under the Creative Commons Attribution-NonCommercial-4.0 International License (CC BY-NC) (http://www.karger.com/Services/OpenAccessLicense), applicable to the online version of the article only. Usage and distribution for commercial purposes requires written permission.
Correspondence to:

Yi Pang, ypang@umc.edu

Abhay Bhatt, abhatt@umc.edu 
epidemiological evidence showed that perinatal infections and associated inflammation are major risk factors for WMI [5-7]. Clinical and experimental evidence suggests that activated microglia play a pivotal role in mediating OPC injury [8-13].

Microglia, the resident immune cells of the central nervous system, can mount innate immune responses upon inflammatory challenges. While such a response is intended to protect the brain, dysregulated microglial activation also leads to injury or developmental disturbance of neighboring neurons and OPCs. Bacterial endotoxin lipopolysaccharide (LPS)-induced damage and developmental disturbances of OPCs are primarily mediated by pro-inflammatory mediators released by activated microglia $[12,13]$. Therefore, approaches to suppress microglial activation and/or inflammation may protect OPCs and ameliorate the burdens of WMI. For example, the tetracycline derivative minocycline has been demonstrated to afford neuroprotection in various models of brain injury by primarily suppressing microglial activation [14]. However, due to its potential adverse effects on development, minocycline has limited therapeutic potential for WMI [15]. As concern on safety, especially longterm effects on neurodevelopment, remains a challenge in developing novel anti-inflammatory agents to fight WMI, drug repurposing may be a viable strategy in this endeavor. In this regard, drugs that have a track record of safety profiles for use in pregnancy should be considered.

Interestingly, macrolides, a group of antibiotics widely used to treat infections in pediatric patients, have been demonstrated as potent anti-inflammatory agents in suppressing various systemic immune cells. Macrolides' most common adverse effects are GI disturbances, elevated transaminases, sensorineural hearing loss, prolonged QTc (in adults and those with underlying heart disease), and most importantly, in neonates, infantile hypertrophic pyloric stenosis $[16,17]$. Among several major classes of macrolides, azithromycin (AZ) appears to be a potent anti-inflammatory agent with better safety profiles [17]. Therefore, we consider AZ an excellent candidate for suppressing microglial activation and protecting the developing brain against WMI. Still, we performed preliminary experiments to compare the effects of 4 major macrolides, clarithromycin, erythromycin, AZ, and roxithromycin, in suppressing pro-inflammatory cytokine IL-6 from LPS-activated microglia. We tested 4 different doses of macrolides $(0.1,0.5,2.5$, and $12.5 \mu \mathrm{g} / \mathrm{mL})$ and measured IL- 6 by enzyme-linked immunosorbent assay (ELISA) to evaluate the dose-response. The preliminary data showed that AZ had a consistent response at all dos- es on suppression of IL-6 compared to other macrolides. Thus, this study focused on AZ.

The immunomodulatory effect of macrolides has been well studied in the treatment of several chronic respiratory conditions and chronic gastritis caused by helicobacter pylori $[18,19]$. Macrolides reduce cytokine production by LPS-stimulated monocytes [20]. Macrolides affect several pathways of the inflammatory process, such as the migration of neutrophils, the oxidative burst in phagocytes, and the production of pro-inflammatory cytokines in monocytes [21-24]. However, it is unknown whether they also inhibit inflammatory responses from microglia. In this study, we first evaluated the effect of AZ on pro-inflammatory cytokines production and nuclear factor $-\kappa \mathrm{B}(\mathrm{NF}-\kappa \mathrm{B})$ activation in LPS-activated microglia and then tested whether AZ protects OPCs against microglia-mediated cytotoxicity and developmental disturbance upon LPS exposure in vitro.

\section{Materials and Methods}

\section{Chemicals and Reagents}

Dulbecco's modified Eagle medium (DMEM)/Ham's F12 and F15 medium, Hank's balanced salt solution (HBSS), fetal bovine serum (FBS), neural basal medium (NBM), B27 supplements, $7.5 \%$ bovine serum albumin (BSA), platelet-derived growth factor, basic fibroblast growth factor, and penicillin/streptomycin: Invitrogen (Carlsbad, CA, USA). Neural Tissue Dissociation kits were obtained from Miltenyi Biotec (Bergisch Gladbach, Germany). Antibodies were obtained from the following sources: neuron-glia antigen 2 (NG2), O4, Olig2, adenomatosis polyposis coli (APC), myelin basic protein (MBP), glial fibrillary acidic protein (GFAP), and CD11b,: Millipore Sigma (St Louis, MO, USA). Iba1: Wako Chemicals (Richmond, VA, USA). Antibodies for NF- $\mathrm{kB}$ p65 and lamin, and nuclei extraction kit: Santa Cruz Biotechnology (Santa Cruz, CA, USA). XTT assay kit: Roche (Indianapolis, IN, USA). TGX Stain-free precast gels and ECL select kits: Bio-Rad Laboratory (Hercules, CA, USA).

\section{Primary Cell Culture}

Preparation of Mixed Glia Culture from Neonatal Rat Brain

The protocols for primary mixed glia, OPCs, and microglia culture were based on our previously described methods [12, 25] but with significant modifications. Since a large quantity of pure microglia is needed in this study, a papain-based tissue dissociation protocol was developed for mixed-glia culture. Our preliminary tests show that this modification results in a significantly higher yield of viable total neural cells and microglia. The process of tissue dissociation was in accordance with the protocol provided with the Neural Tissue Dissociation kit. All procedures for animal care were conducted in accordance with the National Institutes of Health Guide for the Care and Use of Laboratory Animals, and were approved by the Institutional Animal Care and Use Committee at the University of Mississippi Medical Center. Every effort was made to minimize the number of animals used and their suffering. In brief, 1-day-old Sprague Daw- 
ley rat pups were decapitated and their brain cortices were dissected in ice-cold HBSS under a stereomicroscope. The meninges were carefully stripped away and the cortices from 6 pups were chopped into small pieces using a surgical blade in $1 \mathrm{~mL}$ cold HBSS. Next, the tissue was transferred into a $15-\mathrm{mL}$ conical tube, mixed with 1,950 $\mu \mathrm{L}$ of Enzyme A, and gently rotated for $10 \mathrm{~min}$ at $37^{\circ} \mathrm{C}$. After adding $30 \mu \mathrm{L}$ enzyme mix 2 , tissue was rotated for an additional $10 \mathrm{~min}$ at $37^{\circ} \mathrm{C}$. The tissue was then mechanically dissociated by passing through a $1-\mathrm{mL}$ pipette tip for 5 times and rotated again for $10 \mathrm{~min}$. This process was repeated once, after which the dissociated cells were passed through a $40 \mu \mathrm{m}$ strainer. Next, cells were pelleted by centrifugation at $350 \mathrm{~g}$ for $10 \mathrm{~min}$ and resuspended in $10 \mathrm{~mL}$ prewarmed cell culture medium (DMEM/F12 consisting of 10\% FBS). The percentage of viable to dead cells was estimated based on trypan blue staining. Typically, this protocol can yield about $5 \times 10^{8}$ total cells from 6 neonate rats, with $>90 \%$ viability. Cells were maintained in $75-\mathrm{cm}^{2}$ cell culture flasks at $37^{\circ} \mathrm{C}$ in a $\mathrm{CO}_{2}$ incubator.

\section{Isolation of Microglia from Mixed Glial Culture}

The primary cultures consisting of all 3 glial cell types were maintained in DMEM/F12 with $10 \%$ FBS. The medium was changed every 4 th day. About $2 / 3$ of the old medium was replaced with a fresh medium, and precaution was taken not to disturb the loosely attached microglia on the top. Cells reached confluence in about 10-14 days. Many round, phase-bright cells grown on the top of dense cell layers or detached and floated in the medium were mainly microglia. Flasks were gently shaken with hand or strike against the palm 3 days after the last medium change to isolate microglia. The medium was collected in $50 \mathrm{~mL}$ conical tubes and centrifuge at $350 \mathrm{~g}$ for $10 \mathrm{~min}$. The cell pellet was resuspended in $10 \mathrm{~mL}$ fresh medium. If necessary, the process was repeated for a second round after 1 week of culture to obtain more microglia. Based on 6 independent primary cultures, we estimated that at least $5 \times 10^{6}$ microglia could be harvested from mixed glia culture from 6 rat pups. Microglia were plated on poly-L-lysine-coated glass coverslips placed in 12-well plates at a density of $1.5 \times 10^{5}$ cells/coverslip and were maintained in 10\% FBS/DMEM-F12 for $24 \mathrm{~h}$ before experimental treatments. To estimate the purity of microglia, cells on coverslips were fixed with $4 \%$ paraformaldehyde and immunostained with markers for microglia (CD11b and Iba1), astrocytes (GFAP), and OL lineage cells (Olig2). We found that cells were exclusively immunostained with CD11b and Iba1 (Fig. 1a), while very few GFAP- and Olig2-positive cells were detected. The estimated purity of microglia is $>95 \%$.

Isolation and Culture of OPCs

Following isolation of microglia, the flasks were shaken in an orbit shaker at $180 \mathrm{rpm}$ overnight, followed by $200 \mathrm{rpm}$ for $2 \mathrm{~h}$. The medium was collected and filtered through a $40-\mu \mathrm{m}$ cell strainer. Cells were pelleted by centrifugation, resuspended in 10 $\mathrm{mL}$ medium, and transferred to a non-coated $75-\mathrm{cm}^{2}$ flask. After incubation at $37^{\circ} \mathrm{C}$ for $10 \mathrm{~min}$, the flasks were shaken gently by hand to detach loosely attached OPCs, while microglia and astrocytes remained strongly attached to the surface. The supernatant containing predominantly OPCs was centrifuged at $350 \mathrm{~g}$ for 10 min. Cells were resuspended in a 1:1 mixed medium of NBM-B27 and chemically defined medium. Chemically defined medium consists of DMEM/F12, 0.1\% BSA, $100 \mu \mathrm{M}$ putrescine, $20 \mathrm{nM}$ progesterone, $10 \mathrm{nM}$ sodium selenium, $20 \mathrm{nM}$ biotin, $5 \mu \mathrm{g} / \mathrm{mL}$ cysteine, $5 \mathrm{nM}$ hydrocortisone, $5 \mu \mathrm{M}$ insulin, $50 \mu \mathrm{M}$ transferrin, $2 \mathrm{nML}-\mathrm{glu}-$ tamine, and penicillin/streptomycin). Platelet-derived growth factor and basic fibroblast growth factor $(10 \mathrm{ng} / \mathrm{mL}$, each) were included in the medium to promote OPC growth. Cells were passaged 3 times before experimental treatments.

The purity and identification of OPCs were determined by immunocytochemistry using a panel of well-defined antibody markers (i.e., NG2, O4, and Olig2 to identify OPCs, GFAP for astrocytes, and CD-11b/Iba1 for microglia). The estimated purity of OPCs was close to $99 \%$ after 3 generations.

\section{Preparation of Microglia Conditioned Medium and Treatment} of OPCs

Microglia conditioned medium (MCM) was used in 2 experimental settings: for measurement of cytokine levels and treatment of OPCs. To maintain optimal survival and differentiation and eliminate the cofounding effects of serum on OPC differentiation, the serum-free NBM/B27 was used to prepare MCM to study the effects on OPCs. For the MCM used in ELISA detecting pro-inflammatory cytokines, we used DMEM to prepare MCM to avoid potential interference of proteins and chemicals.

To prepare MCM, microglia were washed twice with $1 \times$ HBSS, and $1 \mathrm{~mL}$ of DMEM or NBM/B27 was added into each well of 12well plates. Four experimental groups (each with triplicate wells) were included throughout the study: (1) Control: medium only; (2) AZ alone $(0.5 \mu \mathrm{g} / \mathrm{mL}$ of AZ in medium); (3) LPS + Veh $(1 \mu \mathrm{g} / \mathrm{mL})$; and (4) LPS + AZ (microglia were pretreated with $0.5 \mu \mathrm{g} / \mathrm{mL}$ of AZ for $30 \mathrm{~min}$ before adding $1 \mu \mathrm{g} / \mathrm{mL}$ of LPS $+\mathrm{Veh})$. The MCM was collected at $6,24,48$, and $96 \mathrm{~h}$ following treatment, filtered with a $0.45-\mu \mathrm{m}$ syringe filter, and stored at $-80^{\circ} \mathrm{C}$ until use.

\section{Cell Survival/Death Assay}

OPCs were seeded in a poly-L-lysine-coated 96-well plate at 1.5 $\times 10^{4}$ per well and were incubated overnight. Cells were washed with prewarmed NBM/B27 and treated with MCM for $24 \mathrm{~h}$. Cell survival was quantified by the XTT method following the manufacture's instruction. The optical density (OD) at $492 \mathrm{~nm}$ was acquired by a plate reader (BioTek), and the cell survival rate was calculated as a percentage (\%) of the OD in the treated group over that in the control group, as previously described [12].

\section{Immunocytochemistry}

Cells grown on glass coverslips were rinsed twice with ice-cold PBS and fixed with $4 \%$ paraformaldehyde for $15 \mathrm{~min}$ at room temperature (RT). Following washing in PBS, cells were permeabilized with $0.2 \%$ Triton X-100 and blocked with 5\% normal serum/1\% BSA/0.1\% Triton X-100 in PBS for $1 \mathrm{~h}$. Cells were sequentially incubated with primary antibodies, biotin-conjugated second antibodies, and avidin-conjugated Alexa Fluor 488 or 555, each for 1 $\mathrm{h}$ at RT with 3 washes in PBS. Cells were mounted on slides and viewed under a fluorescence microscope (Olympus BX60). Images were acquired by a monochrome digital camera.

\section{Quantification of OL Differentiation}

To quantify OL differentiation rate, treated cells were doubleimmunostained with NG2 (for OPCs) and APC (for mature OLs). Ten random high power images ( $\times 40$ objectives) were captured for each coverslip, and positive cells were counted manually in a double-blinded fashion. Cell counting from 10 images per coverslip was averaged to represent 1 sample, and 3 samples were included in each experimental group. 


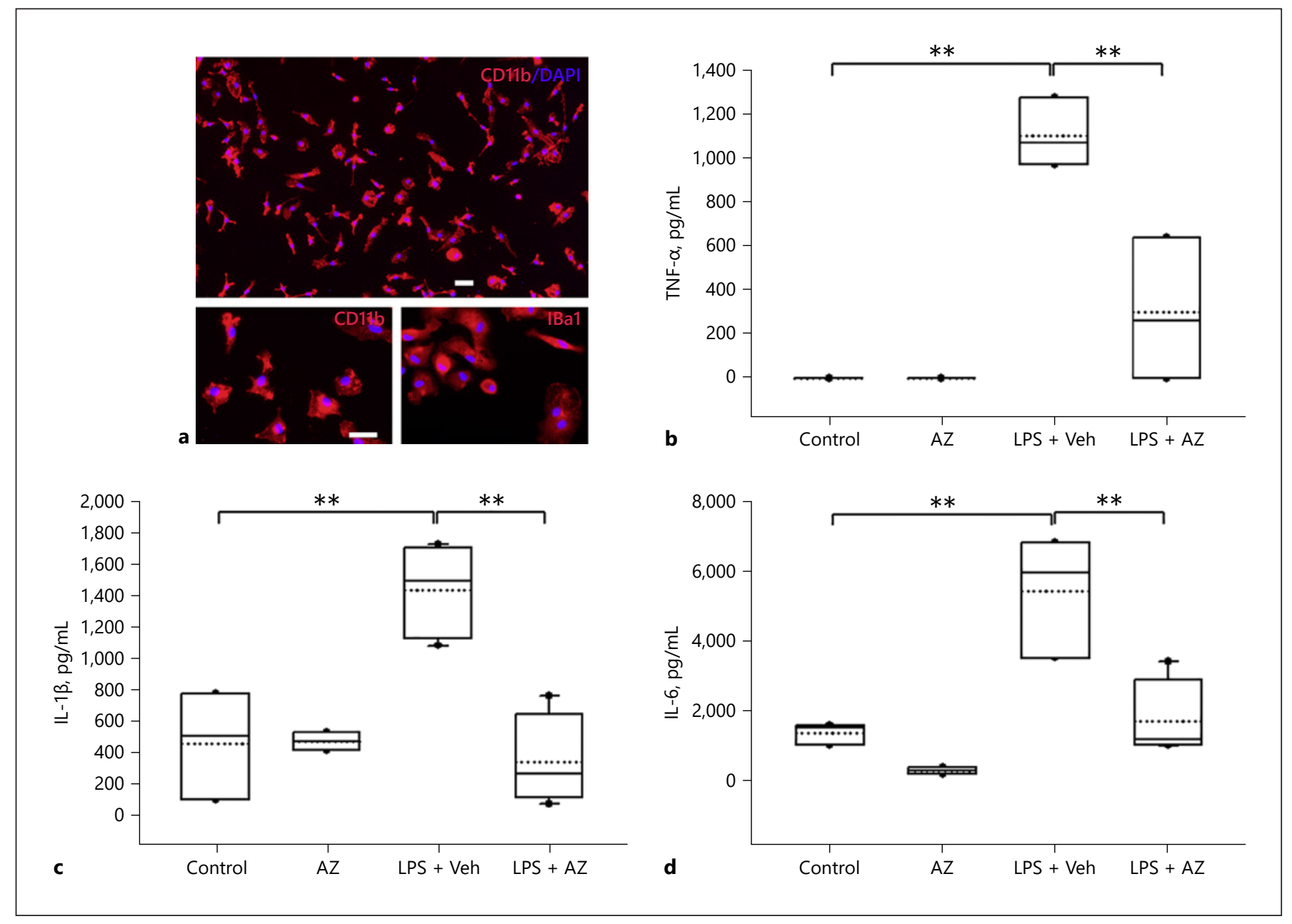

Fig. 1. AZ suppressed pro-inflammatory cytokine release by LPS activated microglia. a Highly enriched microglia were identified by CD11b and IBa1 immunocytochemistry (nuclei were counterstained with DAPI). Scale bars, $50 \mu \mathrm{m}$. TNF- $\alpha$ (b), IL-1 $\beta$ (c), and IL-6 (d) levels in the supernatant of microglia were determined by ELISA following $24 \mathrm{~h}$ of treatment. LPS induced a significant in-

\section{Enzyme-Linked Immunosorbent Assay}

At $24 \mathrm{~h}$ following treatment, MCM was collected to determine levels of IL-1 $\beta$, IL- 6 , and TNF- $\alpha$ by ELISA (R\&D system) following the manufacturer's instruction. Samples were run in duplicates in a $96-$ well plate. Cytokine contents were presented as $\mathrm{pg} / \mathrm{mL}$ medium.

\section{Western Blot}

Cleaved Caspase-3 and MBP in whole-cell extraction of OLs and NF- $\kappa \mathrm{B} / \mathrm{p} 65$ in nuclei fraction of microglia were assessed by Western blot (WB). To prepare whole-cell lysis from OLs, cells were washed once with ice-cold PBS and then detached from the culture surface using a cell scraper. Cells were pelleted by centrifugation, washed twice with ice-cold PBS, and incubated with cell lysis buffer containing $10 \mathrm{mM}$ Tris, $100 \mathrm{mM} \mathrm{NaCl}, 1 \mathrm{mM}$ EDTA, 1 mm EGTA, 1 mM NaF, 20 mM Na4P2O7, 2 mM Na3VO4, crease in all 3 cytokines, and this effect was suppressed by pretreatment with AZ. Box and whiskers represent the median, 25th, 75th percentiles, and 5th, 95th percentiles, respectively. The dotted line represents mean, there were no outliers, ${ }^{*} p<0.05,{ }^{* *} p<0.01, n=$ 3-4. AZ, azithromycin; ELISA, enzyme-linked immunosorbent assay; LPS, lipopolysaccharide.

$1 \%$ Triton X-100, 10\% glycerol, $0.1 \%$ SDS, $0.5 \%$ deoxycholate, $1 \mathrm{mM}$ PMSF, and protease inhibitor cocktail (Sigma) for $30 \mathrm{~min}$ on ice, with vortexes at 10 -min intervals. Microglial nuclei proteins were extracted using the nuclei extraction kit. Total proteins were determined by the BCA kit (ThermoFisher). Samples were denatured and subjected to SDS-PAGE using Bio-Rad TGX stainfree gels, and proteins were transferred to nitrocellulose membranes. The membranes were blocked with $5 \%$ non-fat milk in PBS for $1 \mathrm{~h}$ at RT and incubated with primary antibodies overnight at $4^{\circ} \mathrm{C}$. Following washing, the membranes were incubated with HRP-conjugated secondary antibodies. Signals were detected using ECL select kit. Images were acquired by the ChemiDoc MP Imaging system and data were analyzed by Image Lab software (Bio-Rad). The OD of the target bands was normalized to that of total protein bands, which was acquired prior to developing chemiluminescent signals. 
Statistics

Data were presented as median with the range using box and whisker plots. Differences between groups were tested using twoway ANOVA followed by post hoc Holm-Sidak analysis. $p<0.05$ was considered to be significant. SigmaPlot (version 11) was used (Sigmaplot; Systat Software, Inc., San Jose, CA, USA) for statistical tests.

\section{Results}

\section{AZ Suppressed Pro-Inflammatory Cytokine Release by} LPS-Activated Microglia

TNF- $\alpha$, IL- $1 \beta$, and IL- 6 in MCM were measured at $24 \mathrm{~h}$ after treatment. The effect of LPS depended upon whether microglia were pretreated with $\mathrm{AZ}$, and there were statistically significant interactions between LPS and AZ (two-way ANOVA, F $[1,11]=15.4, p=0.004$, TNF- $\alpha$; $[1,13]=11.2, p=0.007$, IL- $1 \beta ; \mathrm{F}[1,11]=3.9$, $p=0.083$, IL-6). On post hoc analysis, compared to the control, LPS + Veh caused a significant increase in TNF- $\alpha$ $(p<0.001$, Fig. 1 b), IL-1 $\beta$ ( $p=0.001$, Fig. $1 \mathrm{c})$, and IL-6 $(p=0.002$, Fig. $1 \mathrm{~d})$, which were significantly reduced with pretreatment with AZ (LPS + AZ, $p<0.001$, Fig. $1 b-d$ ). There was no difference in TNF- $\alpha$, IL- $1 \beta$, or IL- 6 between control and AZ as well as AZ and LPS + AZ (Fig. 1).

\section{AZ Inhibited NF-Kb Pathway Activation in \\ LPS-Activated Microglia}

NF- $\mathrm{kB} / \mathrm{p} 65$ nuclei translocation was determined by both immunocytochemistry and WB. As shown in the WB in Figure 2a, minimal immunoreactivity of NF- $\kappa \mathrm{B} /$ p65 was detected in the nuclear extracts of the control and $\mathrm{AZ}$ groups. In contrast, a robust increase in NF- $\kappa \mathrm{B} / \mathrm{p} 65$ immunoreactivity was observed following LPS + Veh in a time-dependent manner. Pretreatment of microglia with AZ significantly reduced nuclei NF- $\kappa B / p 65 \mathrm{immu}-$ noreactivity. Quantification of nuclei NF- $\kappa \mathrm{B} / \mathrm{p} 65$ shows that the effect of LPS depended upon whether microglia were pretreated with AZ; there were statistically significant interactions between LPS and AZ (two-way ANO$\mathrm{VA}, \mathrm{F}[1,11]=4.4,8.7,21.4, p=0.07,0.018,0.002$ at 0.5 , 1 and $4 \mathrm{~h}$, respectively). On post hoc analysis, compared to the control, LPS + Veh caused a 2 -fold increase in NF$\kappa \mathrm{B} / \mathrm{p} 65$ at $0.5 \mathrm{~h}(p=0.011)$ and 2.5 -fold increase at $1 \mathrm{~h}$ $(p=0.002)$ and $4 \mathrm{~h}(p<0.001)$. This effect was significantly reduced with pretreatment with AZ (LPS + AZ, $p=0.011, p=0.002, p<0.001$ at $0.5,1$, and $4 \mathrm{~h}$, respectively). There was no significant difference between control and AZ, as well as AZ and LPS + AZ (Fig. 2b). Consistent with the WB data, immunofluorescence staining

Azithromycin Protects OPC from

LPS-Activated Microglia clearly showed that AZ blocked LPS-induced NF- $\mathrm{BB} / \mathrm{p} 65$ translocation from the cytoplasm to the nucleus. As shown in Figure 2c, relatively weak NF- $\kappa B / p 65$ immunoreactivity in the nucleus but strong signals in the cytoplasm was observed in microglia in the control and AZ groups. In contrast, LPS + Veh-treated microglia showed a marked increase of immunoreactivity in the nucleus with a reduction in the cytoplasm. Pretreatment with AZ markedly suppressed LPS-induced NF- $\kappa \mathrm{B}$ translocation to a level comparable to the control.

\section{AZ Protected OPCs against LPS-MCM-Induced Damage}

OPC survival was determined using XTT at 24 and $30 \mathrm{~h}$ following treatments. The results show that the effect of LPS depended upon whether OPC was treated with MCM from AZ-pretreated microglia; there were statistically significant interactions between LPS and AZ (twoway ANOVA, F $[1,31]=15.9,24.7$ at 24 and $30 \mathrm{~h}$, respectively, $p<0.001)$. On post hoc analysis, compared to the control, LPS + Veh reduced XTT at $24(p<0.001)$ and $30 \mathrm{~h}(p<0.015)$; these reductions were significantly prevented with pretreatment with AZ (LPS $+\mathrm{AZ}, p<0.001)$. A minimal increase in XTT was noted following AZ compared to control at $24(p=0.044)$ and $30 \mathrm{~h}(p=0.015)$. There was no difference between AZ and LPS + AZ at $24 \mathrm{~h}$ but a minimal increase in XTT at $30 \mathrm{~h}(p<0.001$, Fig. 3a). Figure $3 b$ shows the quantification of cleaved activated caspase- 3 positive OPCs at $24 \mathrm{~h}$ following treatment with MCM from LPS and AZ-treated microglia. The effect of LPS depended upon whether OPCs were treated with MCM from AZ pretreated microglia; there were statistically significant interactions between LPS and AZ (two-way ANOVA, F $[1,11]=9.5, p=0.015$ ). To further determine whether an increase in OPC survival measured by XTT is primarily due to a reduction in cell death rather than an increase in proliferation, we quantified cleaved caspase- 3 in OPCs by immunofluorescence and WB. Our data show that compared to the control, LPS + Veh significantly increased the number of caspase-3 positive OPCs $(p=0.003)$, which was significantly prevented with pretreatment with AZ (LPS $+\mathrm{AZ}, p=$ 0.001). Consistently, WB demonstrated a marked increase of activated caspase- 3 at $24 \mathrm{~h}$ following treatment with MCM-LPS (Fig. 3c). Quantification of WB data in Figure $3 \mathrm{~d}$ showed statistically significant interactions between LPS and AZ (two-way ANOVA, F $[1,11]=32.2$, $p<0.001)$. On post hoc analysis, compared to the control, LPS + Veh caused a 2.5 -fold increase in activated caspase-3 $(p<0.001)$, which could be significantly prevented 
with pretreatment with AZ (LPS $+\mathrm{AZ}, p<0.001)$. No differences in cleaved activated caspase-3-positive OPCs or activated caspase- 3 protein were noted between control and $\mathrm{AZ}$ as well as AZ and LPS + AZ.
AZ Ameliorated OPC Differentiation Arrest Induced by LPS-Activated Microglia

Next, we investigated whether AZ could prevent OPC differentiation arrest by LPS-activated microglia. The
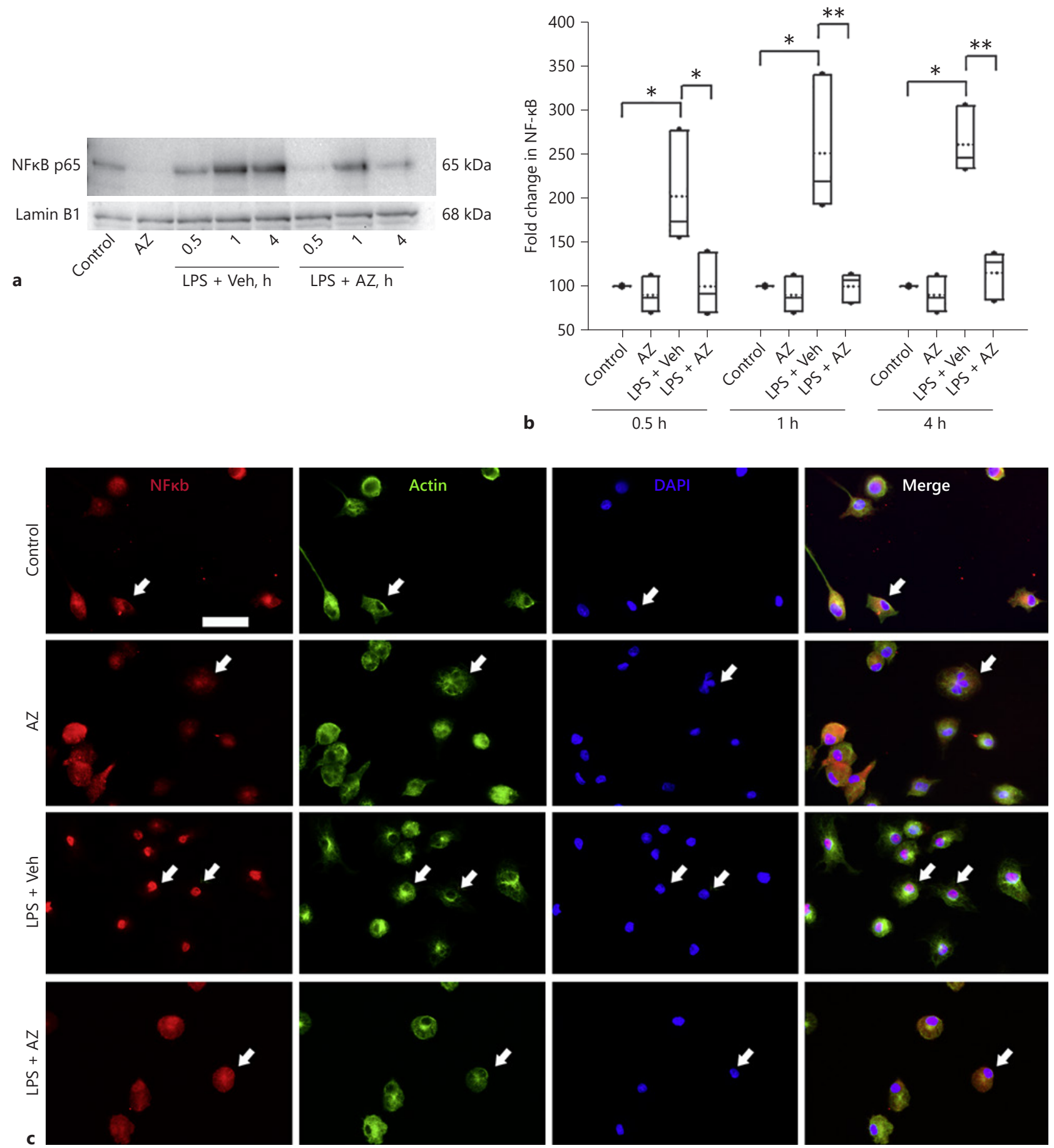

2

(For legend see next page.) 

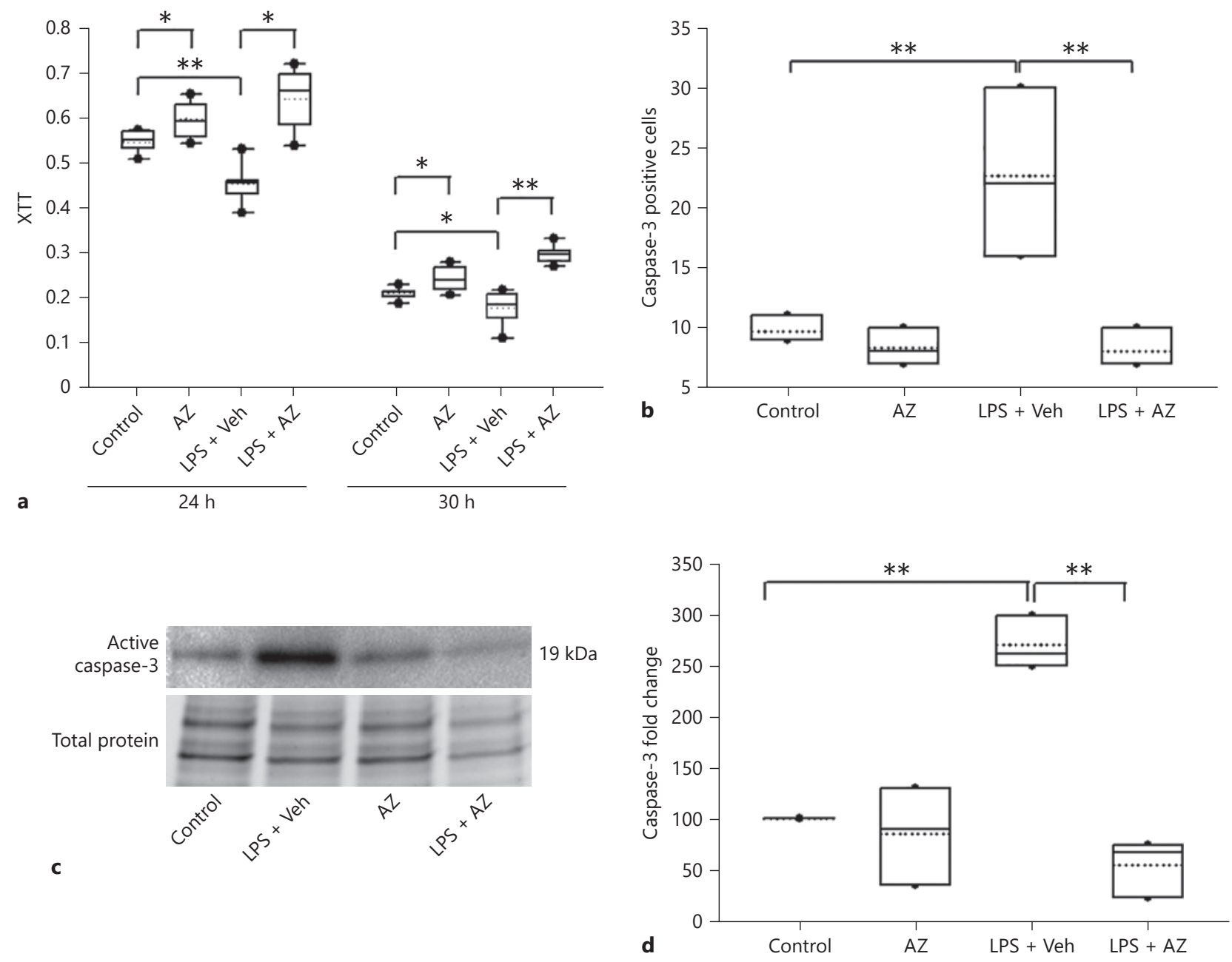

Fig. 3. AZ protected OPCs against LPS-MCM-induced damage. a Quantification of cell survival by XTT at $24 \mathrm{~h}$ and $30 \mathrm{~h}$. b Quantification of active caspase-3+ OPCs at 24 h. c Representative WB of cleaved caspase- 3 and total protein at $24 \mathrm{~h}$. $\mathbf{d}$ The intensities of active caspase- 3 normalized to total proteins are expressed as fold changes relative to the control. Box and whiskers represent the median, 25th, 75th percentiles, and 5th, 95th percentiles, respectively; the dotted line represents mean, there were no outliers, ${ }^{*} p<0.05,{ }^{* *} p<0.01, n=7$. AZ, azithromycin; OPC, oligodendrocyte progenitor cells; LPS-MCM, lipopolysaccharide microglia conditioned medium; WB, Western blot.
Fig. 2. AZ blocked LPS-induced $N F-\kappa B$ activation in microglia. a Representative WB of NF- $\kappa \mathrm{B}$ p65 and Lamin B1 in the nuclear extract. b Quantification of NF- $\mathrm{kB}$ p 65 band intensities as normalized to Lamin B1, and data were presented as fold changes relative to the control. Box and whiskers represent the median, 25th, 75th percentiles, and 5th, 95th percentiles, respectively; the dotted line represents mean, there were no outliers, ${ }^{*} p<0.05,{ }^{* *} p<0.01$, $n=3$. c Representative double-immunofluorescence staining of $\mathrm{NF}-\kappa \mathrm{B}$ p 65 and actin in microglia. In the control, microglial NF- $\kappa \mathrm{B}$ p65 immunostaining is primarily co-localized with actin (cytoplasm, orange) but not DAPI (nuclei). Following LPS treatment, NF- $\kappa$ B p65 immunostaining is predominantly co-localized with DAPI (appears magenta) but not actin, suggesting NF- $\kappa B$ p65 is activated. AZ pretreatment shows a similar NF- $\kappa \mathrm{B}$ p65 immunostaining pattern as that of the control. Arrows indicate co-localization. Scale bar, $50 \mu \mathrm{m}$. AZ, azithromycin; LPS, lipopolysaccharide; $\mathrm{NF}-\kappa \mathrm{B}$, nuclear factor- $\kappa \mathrm{B}$; DAPI, 4',6-diamidino-2-phenylindole; WB, Western blot. 

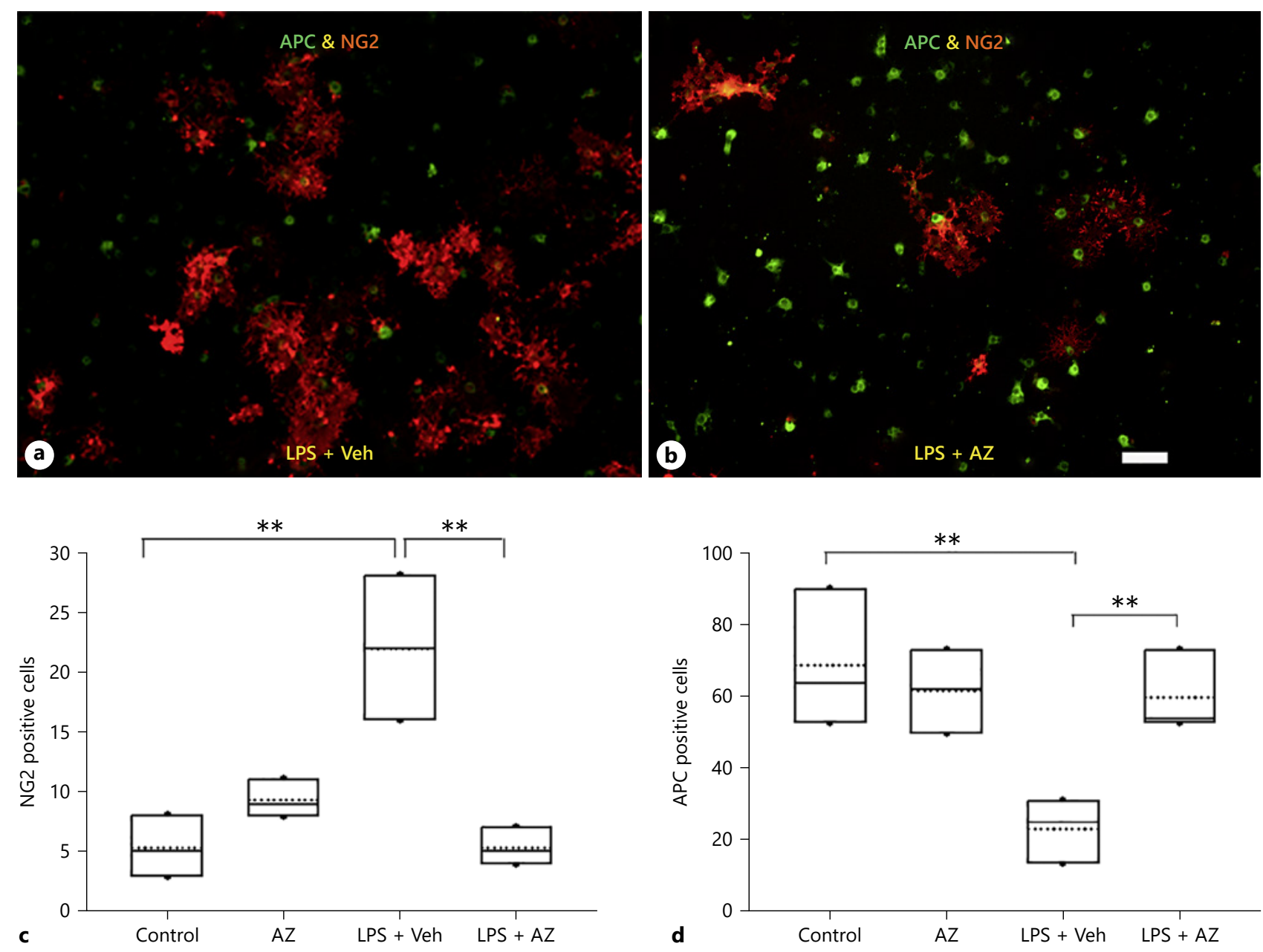

Fig. 4. a, b AZ ameliorated LPS-MCM-induced OL differentiation arrest. OPCs were exposed to a conditioned medium for 5 days and double-immunostained with NG2 and APC. The extent of cell differentiation was determined by counting the number of undifferentiated OPCs ( $\mathrm{NG} 2+$, red) and differentiated mature OLs (APC+, green), which are mutually exclusive. The control culture contains predominantly differentiated APC+ OLs with only a minority of undifferentiated NG2+ OPCs. Exposure to LPS-MCM led to the arrest of OL differentiation as indicated by a higher ratio of NG2+

differentiation of OLs was assessed by quantifying the relative abundance of OPCs (NG2 immunopositive) versus mature OLs (APC immunopositive). As shown in Figure 4, after 5 days of exposure to the MCM, the majority of OPCs in the control cultures differentiated into APC+ mature OLs, leaving only a minority of cells remained as undifferentiated NG2+ OPCs. In contrast, significantly higher numbers of NG2+ cells but lower numbers of APC+ cells were found in LPS-MCM-treat- versus APC+ cells. Treatment with AZ reversed the adverse effect of LPS on OL differentiation. c, $\mathbf{d}$ Box and whiskers represent the median, 25th, 75th percentiles, and 5th, 95th percentiles, respectively; the dotted line represents mean, there were no outliers, ${ }^{*} p<0.05,{ }^{* *} p<0.01, n=3$. Scale bar, $50 \mu \mathrm{m}$. AZ, azithromycin; LPS-MCM, lipopolysaccharide microglia conditioned medium; OPC, oligodendrocyte progenitor cells, OL, oligodendrocyte, NG2, neuron-glia antigen 2, APC, adenomatosis polyposis coli.

ed cultures. This LPS-MCM-mediated OL differentiation arrest was ameliorated by pretreatment with $\mathrm{AZ}$, as shown by a significant increase in APC+ cells and a decrease in NG2+ cells in LPS + AZ group compared to the LPS + Veh group. Statistical analysis shows that the effect of LPS depended upon whether OPCs were treated with MCM from AZ pretreatment; there were statistically significant interactions between LPS and AZ (twoway ANOVA, $\mathrm{F}[1,11]=27.3$ and $8.4, p<0.001$ and $p=$ 


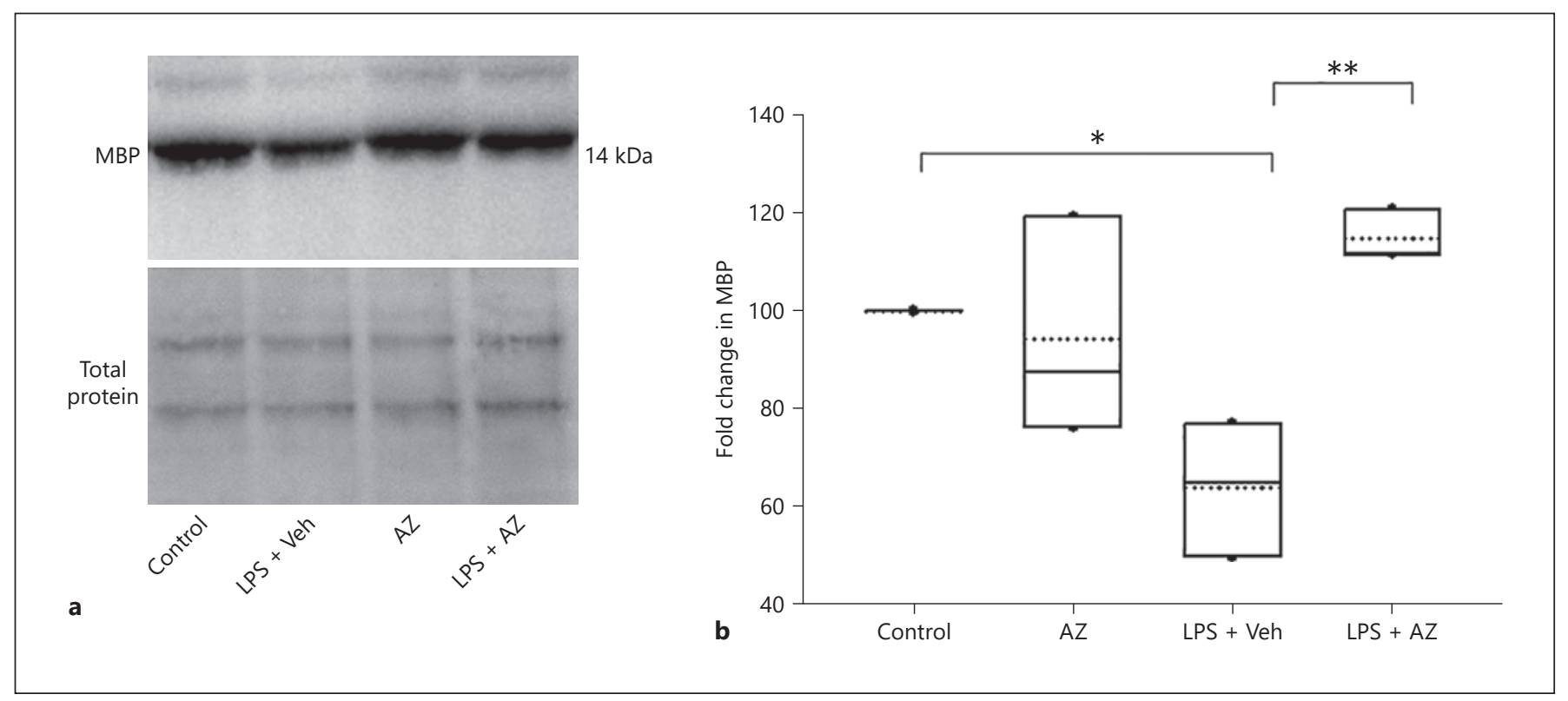

Fig. 5. AZ prevented LPS-MCM-mediated MBP deficit. OPCs were exposed to the conditioned medium for $72 \mathrm{~h}$, and MBP was quantified by WB. a Representative WB of MBP and total proteins (as loading controls). b Total proteins normalized MBP intensity. Data are expressed as fold changes relative to the control. Box and whiskers represent the median, 25th, 75th percentiles, and 5th,

0.020, NG2- and APC-positive cells, respectively). On post hoc analysis, compared to the control, LPS + Veh increased NG2+ cells $(p<0.001)$ and reduced APC+ cells $(p=0.003)$. Pretreatment with AZ (LPS + AZ) reduced NG2+ cells $(p<0.001)$ and increased APC+ cells $(p=0.009)$. No differences in NG2 and APC+ cells were noted between the control and AZ only, as well as AZ and LPS + AZ.

\section{AZ Prevented LPS-MCM-Mediated MBP Deficits in \\ OLs}

To further assess the differentiation and myelination potential of OLs, MBP expression was quantified by WB. As shown in Figure 5, LPS-MCM significantly reduced MBP expression in OPC cultures following a 5-day exposure. LPS-MCM-mediated MBP deficit was prevented by the pretreatment with AZ. Quantification data showed that the effect of LPS depended upon whether OPCs were treated with MCM from AZ pretreated microglia; there were statistically significant interactions between LPS and AZ (two-way ANOVA, F $[1,11]=13.4, p=0.006$ ). On post hoc analysis, compared to the control, LPSMCM exposure significantly reduced MBP protein $(p=$ 0.010). In contrast, pretreatment with $\mathrm{AZ}$ completely 95th percentiles, respectively; the dotted line represents mean, there were no outliers, ${ }^{*} p<0.05,{ }^{* *} p<0.01, n=3$. AZ, azithromycin; LPS-MCM, lipopolysaccharide microglia conditioned medium; MBP, myelin basic protein; OPC, OPC, oligodendrocyte progenitor cells; WB, Western blot.

blocked MBP reduction (LPS $+\mathrm{AZ}, p=0.002$ ). There were no differences between the control and AZ only as well as AZ and LPS + AZ.

\section{Discussion}

The major findings of our study are 2 folds. First, we demonstrate that $\mathrm{AZ}$ is a potent inhibitor of microglial activation that is associated with its ability to block NF- $\kappa B$ activation. Second, AZ is protective against OPC injury and differentiation arrest by LPS-activated microglia.

A large number of studies suggest that OPC injury and developmental disturbance are linked to inflammatory mediators from activated microglia [12, 13, 26-28]; thus, anti-inflammatory drugs have the potential to prevent and/or treat WMI. One of the hurdles in translating basic research finding to clinical therapy in neonatology is the concern that many anti-inflammatory drugs have potential long-term adverse effects on brain development. Therefore, our current study was aimed to identify strong anti-inflammatory reagents that have been extensively used in pediatric patients. AZ is well known for its systemic immunomodulatory effects due to its properties 
like extensive tissue distribution, high accumulation in phagocytes, ability to be delivered at high concentration at infection sites, and suppressive effects on cytokine production $[29,30]$. Evidence suggests that AZ reduces brain infiltration of neutrophils and inflammatory macrophages during infection [30]. Furthermore, AZ has excellent permeability through the blood-brain barrier, as demonstrated by a wide distribution into the brain tissues but not the cerebrospinal fluid following systemic administration [31]. AZ inhibited LPS-induced pregnancy loss in pregnant rats by reducing TNF- $\alpha$ and increasing IL-10 levels [32]. AZ is used to treat various inflammatory diseases such as cystic fibrosis and bronchopulmonary dysplasia because, in addition to antimicrobial effects, AZ has anti-inflammatory effects linked to its ability to suppress NF- $\kappa \mathrm{B}$ activation and TNF- $\alpha$ production $[33,34]$. However, it is not clear whether AZ suppresses microgliamediated neuroinflammation and ameliorates perinatal brain injury. As a first step, we focused on investigating the anti-inflammatory and neuroprotective effects of AZ in cell culture models, which have been used successfully in studying interactions between LPS-activated microglia and OL development [13]. We started with a pilot experiment to determine whether AZ would suppress IL- 6 cytokine in the supernatant from the LPS-stimulated microglia and identify optimal doses. All incremental doses tested $(0.1,0.5,2.5$, and $12.5 \mu \mathrm{g} / \mathrm{mL})$ had a similar inhibitory effect on IL-6. Considering that $0.5 \mu \mathrm{g} / \mathrm{mL}$ was used in other in vitro studies [21,35], this dose was used throughout our study.

Activation of microglia release pro-inflammatory cytokines, especially TNF- $\alpha$, IL- $1 \beta$, and IL- 6 , among others $[9,36,37]$. The inflammatory response in baboons following the Escherichia coli challenge began with an appearance in plasma TNF- $\alpha$, IL-1 $\beta$, followed by a slow but continuous appearance and rise of IL-6 [38]; a similar trend was also noted in human subjects [39]. Previously we showed that these 3 cytokines exhibit distinct temporal profiles in the neonatal rat brain upon LPS challenge, that is, TNF- $\alpha$ and IL- $1 \beta$ start to increase within hours following LPS treatment, while the increase of IL- 6 is relatively delayed [40]. In the current study, we found that LPS-activated microglia produce high levels of TNF- $\alpha$, IL- $1 \beta$, and IL- 6 at $24 \mathrm{~h}$. Conversely, AZ strongly inhibited LPS-induced microglial activation, as demonstrated by significant suppression of TNF- $\alpha$, IL- $1 \beta$, and IL- 6 secretion into the conditioned medium, as well as NF- $\kappa B$ activation in microglia. NF- $\kappa \mathrm{B}$ is one of the major signaling pathways that regulate LPS-mediated microglial activation. NF- $\kappa \mathrm{B}$ exists as an inactive form in the cytoplasm; on stimulation with inflammatory triggering factors, it undergoes nuclear translocation and acts as a transcription factor by binding to regulatory DNA and aids in gene expression and production of pro-inflammatory cytokines. A previous study reported that AZ prevents inflammation-induced activation of NF- $\kappa B$ and subsequent release of IL-6 in tracheal aspirate cells from premature infants [41]. Consistent with this study, our WB data showed a significant increase of NF- $\kappa \mathrm{B}$ in the nuclei fraction upon LPS treatment in a time-dependent manner, and NF- $\kappa B$ translocation from the cytoplasm to the nucleus was clearly visualized by immunofluorescence. Pretreatment with AZ significantly blocked LPS-induced $\mathrm{NF}-\kappa \mathrm{B}$ translocation, as indicated by a predominant retaining of cytoplasmic versus nuclei NF- $\kappa \mathrm{B}$ fluorescence signals. It should be noted that our data do not conclude that blocking NF- $\mathrm{kB}$ is principally responsible for AZmediated anti-inflammatory effect, since other signaling pathways including MAPK-ERK and P38 are also involved in LPS-mediated microglial activation $[42,43]$.

Preterm WMI is a complex sequential process. Increasing evidence suggests that activated microglia play a critical role in the pathogenesis of WMI. Microglia can be activated by both infectious and hypoxic-ischemic insults, 2 major risk factors of WMI. We have previously shown that activated microglia instigate not only OPCs damage but also differentiation arrest, which is considered to be a major mechanism underlying myelination deficits in WMI as well as adult demyelination disorders such as multiple sclerosis. Therefore, anti-inflammatory reagents are also under investigation for multiple sclerosis treatment. For WMI in preterm infants, several drugs targeting neuroinflammation are being studied in animal models [44-47]. For example, it was shown that melatonin promotes OL maturation through its specific receptors [48] with no significant effect on proliferation. Minocycline given immediately after cerebral hypoperfusion promoted OPC proliferation and decreased apoptosis. Though several immunomodulatory therapies are available commercially, their safety and efficacy in preterm neonates, especially on the developing brain, are unknown. In the current study, we found that AZ provides strong protection of OPCs against cytotoxicity by LPS-activated microglia, as indicated by a reduction in activated caspase-3. Such protective effects are likely due to the inhibitory effects of AZ on pro-inflammatory cytokines release. Importantly, we demonstrated that AZ not only prevented LPS-MCM-induced OPC death but also promoted their differentiation. The ultimate goal of therapeutics in WMI is to improve myelination and function- 
al outcomes of preterm infants. OPCs are the predominant OL lineage cells in the embryonic period, and they migrate to the developing white matter and differentiate into mature OLs, which ultimately form the myelin sheath around the axons. Recent human studies indicate that disturbances in OPC maturation rather than cell loss are the principal underlying cause for myelination failure in WMI $[49,50]$. Our study found that AZ treatment prevented LPS-MCM-induced OL lineage progression and downregulation of MBP, suggesting that AZ could restore OL differentiation and potentially myelination ability in an inflammatory environment.

In a recent study using a neonatal rodent model of hypoxic-ischemic brain injury, Barks et al. [51] showed that AZ improved functional and neuropathology outcomes. The authors hypothesized that anti-inflammatory mechanisms were likely involved in AZ-mediated neuroprotection, but those mechanisms were not evaluated in that study. Thus, further in vivo studies using neonatal WMI models are merited to confirm our in vitro findings.

In summary, we demonstrated that $\mathrm{AZ}$ not only reduced pro-inflammatory cytokines release associated with inhibition of the NF- $\kappa \mathrm{B}$ pathway in microglia but also protected OPCs damage and differentiation arrest following exposure to LPS activated microglia. Thus, this proof of concept study provides the first-hand evidence that $\mathrm{AZ}$ and possibly other macrolides may be valuable anti-inflammatory candidates for protecting the developing brain against inflammatory insults.

\section{Statement of Ethics}

This study is approved by our Institutional Animal Care and Use Committee (IACUC), protocol \#1177B.

\section{Conflict of Interest Statement}

The authors declare no conflict of interest.

\section{Funding Sources}

This research was funded by the University of Mississippi Medical Center intradepartmental discovery origination grant.

\section{Author Contributions}

Sumana Ramarao, Kathleen Carter, Yi Pang, and Abhay Bhatt made substantial contributions to conception and design, acquisition of data, and analysis and interpretation of data; Sumana Ramarao, Yi Pang, and Abhay Bhatt contributed to drafting the article and revising it critically for important intellectual content; and Sumana Ramarao, Kathleen Carter, Yi Pang, and Abhay Bhatt approved the final version of the article to be published.

\section{Data Availability Statement}

All data generated or analyzed during this study are included in this article. Further inquiries can be directed to the corresponding author.

\section{References}

1 Volpe JJ. Dysmaturation of premature brain: importance, cellular mechanisms, and potential interventions. Pediatr Neurol. 2019;95: 42-66.

2 Back SA. White matter injury in the preterm infant: pathology and mechanisms. Acta Neuropathol. 2017;134(3):331-49.

3 Volpe JJ. Brain injury in premature infants: a complex amalgam of destructive and developmental disturbances. Lancet Neurol. 2009; 8(1):110-24.

4 Back SA, Luo NL, Borenstein NS, Levine JM, Volpe JJ, Kinney HC. Late oligodendrocyte progenitors coincide with the developmental window of vulnerability for human perinatal white matter injury. J Neurosci. 2001;21(4): 1302-12.

5 Kadhim H, Tabarki B, Verellen G, De Prez C, Rona AM, Sébire G. Inflammatory cytokines in the pathogenesis of periventricular leukomalacia. Neurology. 2001;56(10):1278-84.

6 Yoon BH, Romero R, Yang SH, Jun JK, Kim $\mathrm{IO}$, Choi JH, et al. Interleukin-6 concentra- tions in umbilical cord plasma are elevated in neonates with white matter lesions associated with periventricular leukomalacia. Am J Obstet Gynecol. 1996;174(5):1433-40.

7 Baud O, Emilie D, Pelletier E, Lacaze-Masmonteil T, Zupan V, Fernandez H, et al. Amniotic fluid concentrations of interleukin1beta, interleukin-6 and TNF-alpha in chorioamnionitis before 32 weeks of gestation: histological associations and neonatal outcome. Br J Obstet Gynaecol. 1999;106(1):727.

8 Hofman FM, Hinton DR, Johnson K, Merrill JE. Tumor necrosis factor identified in multiple sclerosis brain. J Exp Med. 1989;170(2): 607-12.

9 Jung YJ, Tweedie D, Scerba MT, Greig NH. Neuroinflammation as a factor of neurodegenerative disease: thalidomide analogs as treatments. Front Cell Dev Biol. 2019;7:313.

10 Agresti C, D'Urso D, Levi G. Reversible inhibitory effects of interferon-gamma and tumour necrosis factor-alpha on oligodendrog- lial lineage cell proliferation and differentiation in vitro. Eur J Neurosci. 1996;8(6): 1106-16.

11 Li J, Baud O, Vartanian T, Volpe JJ, Rosenberg PA. Peroxynitrite generated by inducible nitric oxide synthase and NADPH oxidase mediates microglial toxicity to oligodendrocytes. Proc Natl Acad Sci U S A. 2005;102(28): 9936-41.

12 Pang Y, Cai Z, Rhodes PG. Effects of lipopolysaccharide on oligodendrocyte progenitor cells are mediated by astrocytes and microglia. J Neurosci Res. 2000;62(4):510-20.

13 Pang Y, Campbell L, Zheng B, Fan L, Cai Z, Rhodes P. Lipopolysaccharide-activated microglia induce death of oligodendrocyte progenitor cells and impede their development. Neuroscience. 2010;166(2):464-75.

14 Kovesdi E, Kamnaksh A, Wingo D, Ahmed F, Grunberg NE, Long JB, et al. Acute minocycline treatment mitigates the symptoms of mild blast-induced traumatic brain injury. Front Neurol. 2012;3:111. 
15 Buller KM, Carty ML, Reinebrant HE, Wixey JA. Minocycline: a neuroprotective agent for hypoxic-ischemic brain injury in the neonate? I Neurosci Res. 2009;87(3):599-608.

16 Eberly MD, Eide MB, Thompson JL, Nylund CM. Azithromycin in early infancy and pyloric stenosis. Pediatrics. 2015;135(3):483-8.

17 Smith C, Egunsola O, Choonara I, Kotecha S, Jacqz-Aigrain E, Sammons H. Use and safety of azithromycin in neonates: a systematic review. BMJ Open. 2015;5(12):e008194.

18 Spagnolo P, Fabbri LM, Bush A. Long-term macrolide treatment for chronic respiratory disease. Eur Respir J. 2013;42(1):239-51.

19 Wolter J, Seeney S, Bell S, Bowler S, Masel P, McCormack J. Effect of long term treatment with azithromycin on disease parameters in cystic fibrosis: a randomised trial. Thorax. 2002;57(3):212-6.

20 Labro MT. Anti-inflammatory activity of macrolides: a new therapeutic potential? J Antimicrob Chemother. 1998;41(Suppl B): $37-46$.

21 Ianaro A, Ialenti A, Maffia P, Sautebin L, Rombolà L, Carnuccio R, et al. Anti-inflammatory activity of macrolide antibiotics. J Pharmacol Exp Ther. 2000;292(1):156-63.

22 Mikasa K, Kita E, Sawaki M, Kunimatsu M, Hamada K, Konishi M, et al. The anti-inflammatory effect of erythromycin in zymosaninduced peritonitis of mice. J Antimicrob Chemother. 1992;30(3):339-48.

23 Hand WL, Hand DL, King-Thompson NL. Antibiotic inhibition of the respiratory burst response in human polymorphonuclear leukocytes. Antimicrob Agents Chemother. 1990;34(5):863-70.

24 Takeshita K, Yamagishi I, Harada M, Otomo S, Nakagawa T, Mizushima Y. Immunological and anti-inflammatory effects of clarithromycin: inhibition of interleukin 1 production of murine peritoneal macrophages. Drugs Exp Clin Res. 1989;15(11-12):527-33.

25 Pang Y, Fan LW, Tien LT, Dai X, Zheng B, Cai $Z$, et al. Differential roles of astrocyte and microglia in supporting oligodendrocyte development and myelination in vitro. Brain Behav. 2013;3(5):503-14.

26 Volpe JJ. Systemic inflammation, oligodendroglial maturation, and the encephalopathy of prematurity. Ann Neurol. 2011;70(4):5259.

27 Hagberg H, Mallard C, Ferriero DM, Vannucci SJ, Levison SW, Vexler ZS, et al. The role of inflammation in perinatal brain injury. Nat Rev Neurol. 2015;11(4):192-208.

28 Elovitz MA, Brown AG, Breen K, Anton L, Maubert M, Burd I. Intrauterine inflamma- tion, insufficient to induce parturition, still evokes fetal and neonatal brain injury. Int $J$ Dev Neurosci. 2011;29(6):663-71.

29 Parnham MJ, Erakovic Haber V, Giamarellos-Bourboulis EJ, Perletti G, Verleden GM, Vos R. Azithromycin: mechanisms of action and their relevance for clinical applications. Pharmacol Ther. 2014;143(2):225-45.

30 Lin SJ, Kuo ML, Hsiao HS, Lee PT. Azithromycin modulates immune response of human monocyte-derived dendritic cells and CD4+ T cells. Int Immunopharmacol. 2016; 40:318-26.

31 Jaruratanasirikul S, Hortiwakul R, Tantisarasart T, Phuenpathom N, Tussanasunthornwong S. Distribution of azithromycin into brain tissue, cerebrospinal fluid, and aqueous humor of the eye. Antimicrob Agents Chemother. 1996;40(3):825-6.

32 Er A. Azithromycin prevents pregnancy loss: reducing the level of tumor necrosis factoralpha and raising the level of interleukin-10 in rats. Mediators Inflamm. 2013;2013:928137.

33 Stellari FF, Sala A, Donofrio G, Ruscitti F, Caruso $\mathrm{P}$, Topini TM, et al. Azithromycin inhibits nuclear factor-kappaB activation during lung inflammation: an in vivo imaging study. Pharmacol Res Perspect. 2014;2(5):e00058.

34 Cigana C, Assael BM, Melotti P. Azithromycin selectively reduces tumor necrosis factor alpha levels in cystic fibrosis airway epithelial cells. Antimicrob Agents Chemother. 2007; 51(3):975-81.

35 Dreses-Werringloer U, Padubrin I, Zeidler $\mathrm{H}$, Köhler L. Effects of azithromycin and rifampin on Chlamydia trachomatis infection in vitro. Antimicrob Agents Chemother. 2001;45(11):3001-8.

36 Puffenbarger RA, Boothe AC, Cabral GA. Cannabinoids inhibit LPS-inducible cytokine mRNA expression in rat microglial cells. Glia. 2000;29(1):58-69.

37 Sheng WS, Hu S, Kravitz FH, Peterson PK, Chao CC. Tumor necrosis factor alpha upregulates human microglial cell production of interleukin-10 in vitro. Clin Diagn Lab Immunol. 1995;2(5):604-8.

38 Creasey AA, Stevens P, Kenney J, Allison AC, Warren K, Catlett R, et al. Endotoxin and cytokine profile in plasma of baboons challenged with lethal and sublethal Escherichia coli. Circ Shock. 1991;33(2):84-91.

39 van Deventer SJ, Büller HR, ten Cate JW, Aarden LA, Hack CE, Sturk A. Experimental endotoxemia in humans: analysis of cytokine release and coagulation, fibrinolytic, and complement pathways. Blood. 1990;76(12): $2520-6$.
40 Pang Y, Cai Z, Rhodes PG. Disturbance of oligodendrocyte development, hypomyelination and white matter injury in the neonatal rat brain after intracerebral injection of lipopolysaccharide. Brain Res Dev Brain Res. 2003;140(2):205-14.

41 Aghai ZH, Kode A, Saslow JG, Nakhla T, Farhath S, Stahl GE, et al. Azithromycin suppresses activation of nuclear factor-kappa B and synthesis of pro-inflammatory cytokines in tracheal aspirate cells from premature infants. Pediatr Res. 2007;62(4):483-8.

42 Qin S, Yang C, Huang W, Du S, Mai H, Xiao J, et al. Sulforaphane attenuates microgliamediated neuronal necroptosis through down-regulation of MAPK/NF- $\mathrm{kB}$ signaling pathways in LPS-activated BV-2 microglia. Pharmacol Res. 2018;133:218-35.

43 Jo SH, Kim ME, Cho JH, Lee Y, Lee J, Park YD, et al. Hesperetin inhibits neuroinflammation on microglia by suppressing inflammatory cytokines and MAPK pathways. Arch Pharm Res. 2019;42(8):695-703.

44 Cheng S, Hou J, Zhang C, Xu C, Wang L, Zou $\mathrm{X}$, et al. Minocycline reduces neuroinflammation but does not ameliorate neuron loss in a mouse model of neurodegeneration. Sci Rep. 2015;5(1): 10535 .

45 Huo Y, Rangarajan P, Ling EA, Dheen ST. Dexamethasone inhibits the Nox-dependent ROS production via suppression of MKP1-dependent MAPK pathways in activated microglia. BMC Neurosci. 2011;12(1):49.

$46 \mathrm{YuX}$, Li Z, Zheng H, Ho J, Chan MT, Wu WK. Protective roles of melatonin in central nervous system diseases by regulation of neural stem cells. Cell Prolif. 2017;50(2):e12323.

47 Pathipati P, Ferriero DM. The differential effects of erythropoietin exposure to oxidative stress on microglia and astrocytes in vitro. Dev Neurosci. 2017;39(1-4):310-22.

48 Olivier P, Fontaine RH, Loron G, Van Steenwinckel J, Biran V, Massonneau V, et al. Melatonin promotes oligodendroglial maturation of injured white matter in neonatal rats. PLoS One. 2009;4(9):e7128.

49 van Tilborg E, de Theije CGM, van Hal M, Wagenaar N, de Vries LS, Benders MJ, et al. Origin and dynamics of oligodendrocytes in the developing brain: implications for perinatal white matter injury. Glia. 2018;66(2):221-38.

50 Back SA, Miller SP. Brain injury in premature neonates: a primary cerebral dysmaturation disorder? Ann Neurol. 2014;75(4):469-86.

51 Barks JDE, Liu Y, Wang L, Pai MP, Silverstein FS. Repurposing azithromycin for neonatal neuroprotection. Pediatr Res. 2019;86(4): 444-51. 